\title{
Carbamazepine-induced toxic epidermal necrolysis in a patient despite testing negative for HLA $B * 15: 02$ allele
}

\section{Dear Editor,}

Carbamazepine (CBZ) has been used in Singapore since $1988^{1}$ as an effective drug for the treatment of conditions such as epilepsy, neuropathy, neuralgia and psychiatric conditions. However, it is a high-risk drug for Stevens-Johnson syndrome/toxic epidermal necrolysis (SJS/TEN), particularly in Asians, and in 2004, a genetic marker human leukocyte antigen (HLA)-B*1502 in Han Chinese was identified. ${ }^{2}$ In Singapore, these findings were validated and the carriage of HLA-B*1502 was associated with CBZinduced SJS/TEN (odds ratio 181, $P<0.0001$ ). ${ }^{3}$ This led to the recommendations for HLA-B*1502 genotyping prior to initiation of $\mathrm{CBZ}$ in new patients of Asian ancestry as standard of care, to reduce the risk of developing SJS/TEN. ${ }^{3}$

Through this implementation, there has been more than 1,000 tests performed annually, and the number of reported cases of SJS/TEN associated with CBZ has decreased sharply by $>90 \%$ after policy implementation. ${ }^{4}$

We report a case of a patient who was given CBZ and developed TEN despite testing negative for the HLA B*15:02 allele. This case serves to remind clinicians that a negative test result for the HLA B*1502 allele-while making it less likely_does not preclude the possibility of SJS/TEN development in a patient prescribed with $\mathrm{CBZ}$, and that early recognition of this syndrome is important given its significant morbidity and mortality.

A 27-year-old Chinese man with a history of autism spectrum disorder-previously prescribed with sodium valproate $\mathrm{CR} 750 \mathrm{mg} \mathrm{ON}$, fluoxetine $40 \mathrm{mg} \mathrm{OM}$, and risperidone $2 \mathrm{mg} \mathrm{BD}$, clonazepam $1 \mathrm{mg}$ TDS - presented with worsening aggression and violent behaviour. He was seen by his psychiatrist who then recommended CBZ for treatment of his aggressive behaviour. Prior to initiation of $\mathrm{CBZ}$, he underwent a serum polymerase chain reaction test for HLA-B*15:02 allele and tested negative.

On the 11th day after starting CBZ, he developed a fever. His fever persisted for 3 days despite empirical treatment with antibiotics. Given mild derangements in his liver function tests, an infective aetiology was suspected and he underwent extensive investigations, including computed tomography scan of his brain, chest, abdomen and pelvis, and a transthoracic echocardiogram that did not reveal any sources of sepsis or occult infection. Viral hepatitis markers, atypical bacterial infections (e.g. Mycoplasma spp.) and autoimmune markers were also screened and showed no abnormalities. Four days after the onset of fever, he started developing erythematous macules on his trunk, upper thighs and arms. A dermatology consult was made and a diagnosis of TEN was considered. A skin biopsy was immediately performed. Several of his psychiatric medications, including CBZ, were stopped due to their possible implications as a culprit drug.

His skin biopsy was reported as vacuolar interface changes associated with numerous apoptotic bodies at the dermal-epidermal junction. At this point, his rash progressed rapidly with the formation of flaccid blisters and erosions, along with conjunctival and oral mucosal involvement. Nikolsky's sign was positive with body surface area (BSA) of 50\%, and a diagnosis of TEN was made. His SCORe of Toxic Epidermal Necrolysis (SCORTEN) was 2 at the time of diagnosis, due to the presence of tachycardia, and the affected BSA being $>10 \%$. He was started on supportive treatment, and cyclosporine A was also initiated at a dose of $1 \mathrm{mg} / \mathrm{kg} /$ day intravenously.

A detailed review of his exposure to new drugs in the recent 3 months prior to the onset of TEN was made, and a few potential culprits were identified, namely CBZ, zuclopenthixol, co-ping was performed again, which was negative. An extended HLA typing was also done and the results are in Table 1.

In the subsequent days, the patient remained persistently febrile and tachycardic, with progression of his involved BSA to $80-90 \%$ (Fig. 1). He was managed with meticulous supportive care with intravenous fluids, regular scheduled dressing changes using non-adherent dressings, and an ongoing infusion of cyclosporine $70 \mathrm{mg}$ once daily. He remained clinically stable although initial improvement in his cutaneous and mucosal involvement was marginal.

However, 6 days after he was transferred to the burns unit, he developed type 1 respiratory failure. He responded initially to doses of furosemide as treatment 
Table 1. Results of extended HLA typing: none matched existing reports of alternative HLA alleles associated with higher risk of CBZ-induced TEN

\begin{tabular}{ll}
\hline HLA typing (using sequence-based typing) & Results \\
\hline HLA A & $\begin{array}{l}A^{*} 02: 07 \\
\\
\end{array}$ \\
HLA B & $\mathrm{B}^{*} 27: 01 / 295 / 303 / 324 / 328 / 353$ \\
& $\mathrm{~B}^{*} 40: 01 / 72 / 124 / 183 / 353 / 379 / 386 / 416 / 417 / 431 / 439 / 443$ \\
HLA C & $\mathrm{C}^{*} 04: 01 / 29 / 30 / 33 / 114 / 172 / 275 / 277 / 320 / 375 / 380 / 391$ \\
& $\mathrm{C} * 12: 02 / 41 / 72 / 132 / 146 / 228 / 261 / 285 / 304$ \\
\hline
\end{tabular}

CBZ: carbamazepine; HLA: human leukocyte antigen; TEN: toxic epidermal necrolysis

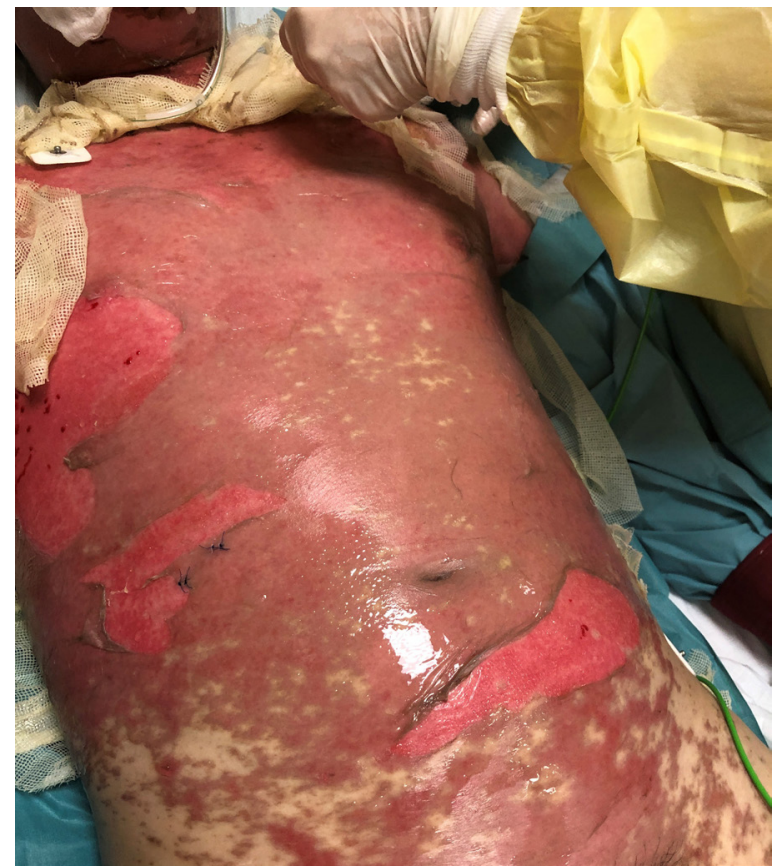

Fig. 1. Extensive denudation of the entire trunk and upper limbs, with some areas of flaccid blisters formation on the lower abdomen.

for fluid overload but rapidly deteriorated overnight and eventually died from cardiac arrest.

The majority of CBZ-induced SJS/TEN in Han Chinese and Southeast Asians are associated with HLA-B*1502. Initial studies on CBZ-induced SJS/TEN in Taiwan and Singapore reported that the negative predictive value of HLA-B*1502 was from 99.9$100 \% .^{5}$ A subsequent study based on a larger population group in Taiwan demonstrated that while HLA-B*1502 was significantly associated with a higher risk of SJS/TEN-almost 100 times higher than for a non-carrier - the negative predictive value was lower at $92 \%{ }^{6}$
In light of these findings and our case report, there are a few practical implications for patients of Asian descent in Singapore. First, regardless of testing negative for HLA B*1502, patients who are to be initiated on CBZ should still be counselled on the low risk of developing SJS/TEN.

Second, it must also be recognised that HLA-B*1502 is only a genetic marker for SJS/TEN. It does not predict other severe adverse drug reactions such as drug reaction with eosinophilia and systemic symptoms, ${ }^{6}$ which is also highly associated with CBZ and other anti-epileptics. ${ }^{7}$ Therefore, it is essential that all patients newly initiated on CBZ are closely monitored and counselled to watch for reactions even if they are HLA-B*1502 negative.

In our patient, we sought to review further other HLA alleles that might have rendered him susceptible to developing CBZ-induced TEN. A review of literature has shown some associations with HLA-A*31:01, HLA $B * 15: 11$, HLA B*15:18, HLA-B*59:0. ${ }^{8-10}$ We proceeded with an extended testing for HLA A, $\mathrm{B}$ and $\mathrm{C}$ alleles, but none corresponded to existing risk markers. As more of such HLA B-negative SJS/TEN cases are identified, genotyping should be performed to identify other potential risk markers, although its cost-effectiveness is debatable. Such markers, if present, might further refine future genetic screening tests in addition to tests for HLA-B*1502.

\section{REFERENCES}

1. Health Sciences Authority, Singapore. Recommendations for HLA-B*1502 genotype testing prior to initiation of carbamazepine in new patients, 29 August 2013. Available at: https://www.hsa.gov. sg/announcements/safety-alert/recommendations-for-hla-b-1502genotype-testing-prior-to-initiation-of-carbamazepine-in-new-patients. Accessed on 12 October 2020. 
2. Chung WH, Hung SI, Hong HS, et al. Medical genetics: a marker for Stevens-Johnson syndrome. Nature 2004;428:486.

3. Health Sciences Authority, Singapore. HLA-B*1502 genotype testing: Towards safer use of carbamazepine, 31 December 2013. Available at: https://www.hsa.gov.sg/announcements/safety-alert/ hla-b-1502-genotype-testing-towards-safer-use-of-carbamazepine. Accessed on 12 October 2020.

4. Sung C, Tan L, Limenta M, et al. Usage Pattern of Carbamazepine and Associated Severe Cutaneous Adverse Reactions in Singapore Following Implementation of HLA-B*15:02 Genotyping as Standard-of-Care. Front Pharmacol 2020;11:527.

5. Ferrell PB Jr, McLeod HL. Carbamazepine, HLA-B*1502 and risk of Stevens-Johnson syndrome and toxic epidermal necrolysis: US FDA recommendations. Pharmacogenomics 2008;9:1543-6.

6. Hsiao YH, Hui RC, Wu T, et al. Genotype-phenotype association between HLA and carbamazepine-induced hypersensitivity reactions: strength and clinical correlations. J Dermatol Sci 2014;73:101-9.

7. Kardaun SH, Sekula P, Valeyrie-Allanore L, et al. Drug reaction with eosinophilia and systemic symptoms (DRESS): an original multisystem adverse drug reaction. Results from the prospective RegiSCAR study. Br J Dermatol 2013;169:1071-80.
8. Fan WL, Shiao MS, Hui RC, et al. HLA Association with DrugInduced Adverse Reactions. J Immunol Res 2017;2017:3186328.

9. Pavlos R, Mallal S, Ostrov D, et al. Fever, rash, and systemic symptoms: understanding the role of virus and HLA in severe cutaneous drug allergy. J Allergy Clin Immunol Pract 2014; 2:21-33

10. Phillips EJ, Sukasem C, Whirl-Carrillo M, et al. Clinical Pharmacogenetics Implementation Consortium Guideline for HLA Genotype and Use of Carbamazepine and Oxcarbazepine: 2017 Update. Clin Pharmacol Ther 2018;103:574-81.

Bochao Jiang, ${ }^{1}{ }_{M B B S}$, Choon Chiat $\underline{\mathrm{Oh}},{ }^{1}{ }_{M R C P}$, Ki Wei Tan, ${ }^{2}{ }_{M R C P}$, Haur Yueh Lee, ${ }^{1}$ MCRP

${ }^{1}$ Department of Dermatology, Singapore General Hospital, Singapore

${ }^{2}$ Department of Dermatology, Changi General Hospital, Singapore

Correspondence: Dr Haur Yueh Lee, Department of Dermatology, Singapore General Hospital, Outram Road, Singapore 169608.

Email: lee.haur.yueh@singhealth.com.sg 